\title{
First Case of Small Bowel Cancer in a Post Roux-en-Y Gastric Bypass Weight-Loss Surgery: A Case Report
}

\author{
Abraham Yacoub $^{\mathrm{a}}$, Leslie Bank ${ }^{\mathrm{b}, \mathrm{d}}$, Jagmohan S. Sidhu ${ }^{\mathrm{c}}$
}

\begin{abstract}
Obesity is currently one of the most common chronic diseases and has become a major health concern in Western countries. Consequently, rise of bariatric surgery nationwide, has been increasing. The Rouxen-Y gastric bypass (RYGB) is a type of surgery indicated in the treatment of morbid obesity. At our institution, we report a case of a 48-year-old female with a history of morbid obesity, who underwent RYGB 13 years ago for weight loss, and was admitted to the hospital for abdominal pain. Upper endoscopy then revealed small bowel cancer.
\end{abstract}

Keywords: Small bowel cancer; Roux-en-Y procedure; Morbid obesity

\section{Introduction}

Obesity is a major public health problem in the United States [1]. Bariatric surgery, including laparoscopic Roux-en-Y gastric bypass (LRYGB), has been shown to be effective in achieving significant long-term weight loss [2]. Malignant small bowel tumors are perhaps the most devastating gastrointestinal (GI) malignancies due to difficulty with diagnosis [3]. We present a case of a 48-year-old female with a history of morbid obesity who underwent RYGB for weight loss and who developed small bowel cancer years later.

Manuscript accepted for publication January 05, 2015

aUnited Health Services Wilson Medical Center, 33-57 Harrison St., Johnson City, NY, USA

${ }^{b}$ Resident Research and Infusion Services, United Health Services Wilson Medical Center, Upstate Medical University, Binghamton Gastroenterology Associates, 40 Mitchell Ave., Binghamton, NY 13903, USA

'Anatomic Pathology \& Clinical Pathology, Hematology, United Health Services Wilson Medical Center, 33-57 Harrison St., Johnson City, NY, USA

${ }^{\mathrm{d} C}$ Corresponding Author: Leslie Bank, United Health Services Wilson Medical Center, Upstate Medical University, Binghamton Gastroenterology Associates, 40 Mitchell Ave., Binghamton, NY 13903, USA.

Email: leslie.bank@gmail.com

doi: http://dx.doi.org/10.14740/jmc2027w

\section{Case Report}

On October 25, 2013, a 48-year-old female with a medical history of morbid obesity and status post RYGB performed 13 years ago was evaluated in the emergency department for worsening postprandial abdominal pain, nausea, and vomiting. The patient lost over 200 pounds after the RYGB procedure. However, recently she has gained over 50 pounds. She denied any fever, chills, or sweats. The patient had a family history of colon cancer.

In 2011, she was admitted to the hospital with similar symptoms and an EGD was performed. During the procedure, the scope was passed through the small intestines to a distance of $60 \mathrm{~cm}$ distal to the gastroduodenal anastomosis. No pathology was visualized.

The patient's vital signs were unremarkable. Physical examination revealed a distended abdomen with epigastric tenderness and a succussion splash was heard. Laboratory data showed normocytic anemia and mild hypocalcemia. The patient was admitted for conservative management of small bowel obstruction. Subsequently, gastroenterology was consulted

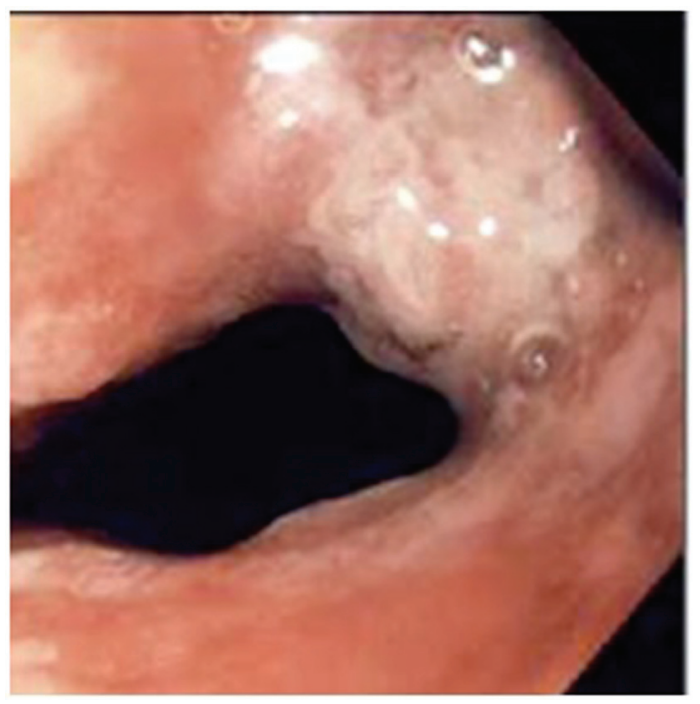

Figure 1. Olympus videogastroscope shows a friable lesion in the Roux-en-Y gastric bypass limb at about $80 \mathrm{~cm}$ distal to the gastroduodenal anastomosis. 


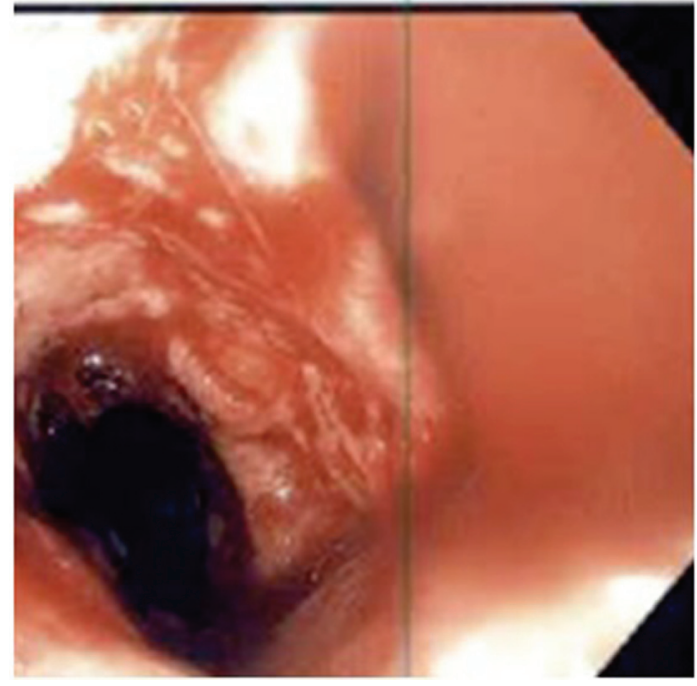

Figure 2. The oozing of blood is due to its friability after the scope was passed through.

and EGD was performed, which revealed a lesion in the RYGB limb at about $80 \mathrm{~cm}$ distal to the gastroduodenal anastomosis (Fig. 1, 2). Three days after her hospital admission, she was taken for a diagnostic laparoscopy with conversion to open laparotomy and underwent resection of a portion of Rou-en-Y limb with partial omentectomy. Surgical resection of the small bowel tumor revealed a $5.2 \times 2.4 \mathrm{~cm}$ centrally ulcerated and erythematous mass with slightly raised polypoid edges (Fig. 3, 4). Biopsies revealed invasive glands and cribiform structures composed of columnar cells with hyperchromatic nuclei that have lost normal polarity (Fig. 5). Two out of the 20 lymph nodes were positive for metastatic disease.

Three months following the small bowel cancer surgical resection, the patient came in to the gastroenterology clinic for follow-up. She complained of nausea, due to the chemotherapy treatment. A repeat $\mathrm{CT}$ scan of the abdomen, pelvis, and chest did not show any metastatic lesion.

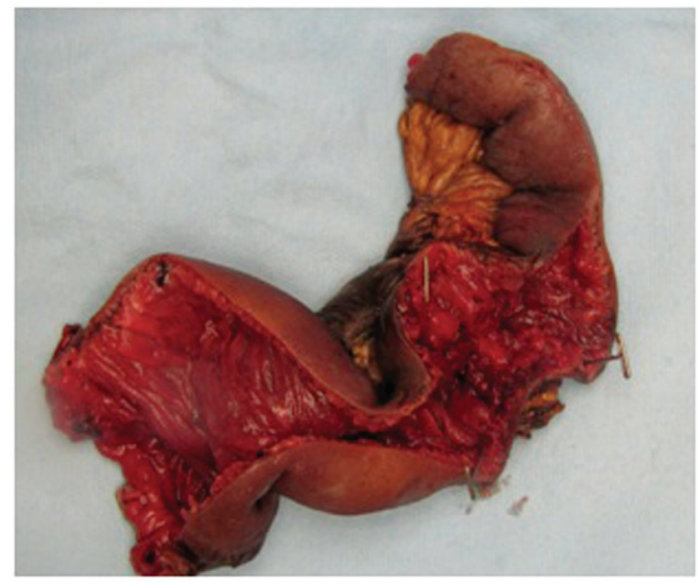

Figure 3. Resected specimen of small intestine that has been opened longitudinally along the antimesenteric border and shows a centrally ulcerated and erythematous mass with slightly raised polypoid edges.

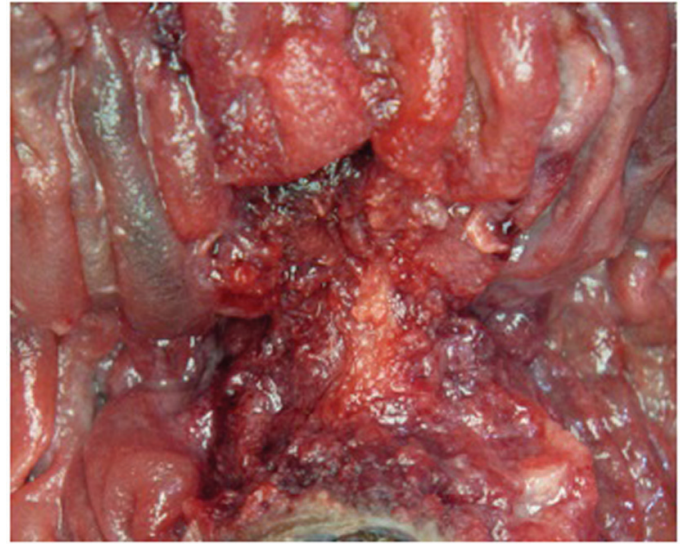

Figure 4. Closer view of the resected specimen of small intestine showing a centrally ulcerated and erythematous mass with slightly raised polypoid edges.

She received folinic acid, fluorouracil, and irinotecan (FOLFIRI) chemotherapy regimen with a total of 14 cycles from December 2013 to October 2014 complicated by fatigue, extensive lower extremity lymphedema, and neuropathy.

\section{Discussion}

Malignant tumors of the small intestine are among the rarest types of GI cancers and adenocarcinoma is the most common histologic type of small bowel cancer [4]. However, very little is known about its etiology. Several behavioral risk factors include consumption of red or smoked meat, saturated fat, obesity, and smoking [5].

One interesting study by Derogar et al concluded that obesity surgery seems to be associated with an increased risk of colorectal cancer overtime [6]. The association between small bowel cancer and bariatric surgery is unknown. Nevertheless,

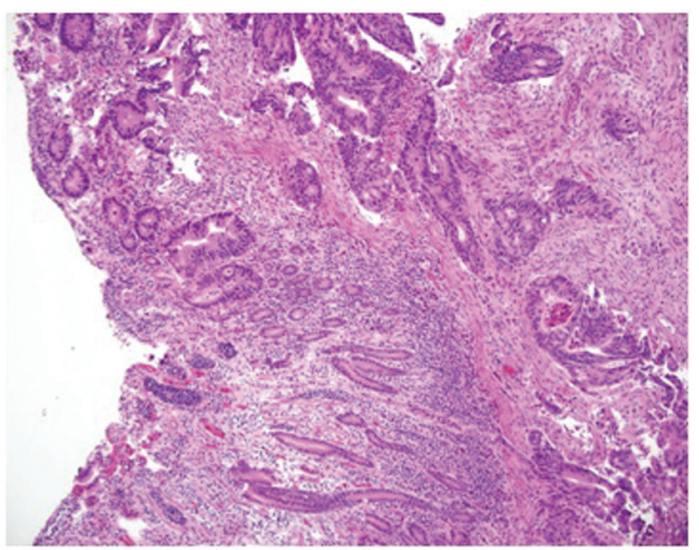

Figure 5. Hematoxylin and eosin (H\&E) stain of the friable small bowel lesion at $\times 100$. Left lower half of the picture shows normal small intestinal mucosa. Rest of the picture shows invasive adenocarcinoma, i.e. invasive glands and cribriform structures composed of columnar cells with hyperchromatic nuclei that have lost normal polarity. Invasive adenocarcinoma is surrounded by desmoplastic (fibrotic) reaction. 
some cases were still documented $[7,8]$. We believe our case of small bowel cancer in bariatric surgery for weight loss is the first to be reported. Therefore, further studies need to be done to confirm if there is an association between small bowel cancer and bariatric surgery.

The most common presenting symptoms are abdominal pain and weight loss, followed by nausea and vomiting [9]. Double-balloon enteroscopy is a novel diagnostic procedure in the evaluation of the small bowel which is performed via oral and anal approach [10]. Our patient had complained of similar symptoms 2 years ago. An EGD procedure was carried out but unfortunately we failed to pass the scope far enough through the small bowel.

The basic treatment for cancer of the small intestine is surgical resection. Palliative surgery and chemotherapy are considered in cases where resection is not possible or the cancer recurs [11]. Currently, the best evidence favors the use of fluorouracil plus a platinum agent for adjuvant chemotherapy and capecitabine and irinotecan for palliative chemotherapy [12]. Folinic acid, fluorouracil, and oxaliplatin (FOLFOX) can also be used [13]. FOLFOX regimen was not administered due to the neuropathy and lymphedema side effects in which our patient had.

\section{Conclusion}

Adenocarcinoma of the small bowel is a rare and deadly cancer. Clinicians who encounter patients admitted to the hospital for abdominal pain, nausea, and vomiting with a previous history of RYGB procedure for weight loss, should fully examine the small bowel to rule out serious pathologies, such as cancer.

\section{References}

1. Baskin ML, Ard J, Franklin F, Allison DB. Prevalence of obesity in the United States. Obes Rev. 2005;6(1):5-7.

2. Lee WJ, Ser KH, Lee YC, Tsou JJ, Chen SC, Chen JC. Laparoscopic Roux-en-Y vs. mini-gastric bypass for the treatment of morbid obesity: a 10-year experience. Obes
Surg. 2012;22(12):1827-1834.

3. Ashley SW, Wells SA, Jr. Tumors of the small intestine. Semin Oncol. 1988;15(2):116-128.

4. Vagholkar K, Mathew T. Adenocarcinoma of the small bowel: a surgical dilemma. Saudi J Gastroenterol. 2009; 15(4):264-267.

5. Pan SY, Morrison H. Epidemiology of cancer of the small intestine. World J Gastrointest Oncol. 2011;3(3):33-42.

6. Derogar M, Hull MA, Kant P, Ostlund M, Lu Y, Lagergren J. Increased risk of colorectal cancer after obesity surgery. Ann Surg. 2013;258(6):983-988.

7. Choda Y, Ninomiya M, Fujiwara Y, Kanazawa T, Harano M, Matsukawa H, Ojima Y, et al. [A case report of multiple adenocarcinoma in small intestine after total gastrectomy by Roux-en-Y re-construction]. Gan To Kagaku Ryoho. 2011;38(12):2042-2044.

8. Ninomiya R, Sakamoto H, Suto Y, Obuchi Y, Yatsuoka T, Nishimura Y, Kawashima Y, et al. [A case of small intestinal cancer in the efferent loop of roux-en Y reconstruction after total gastrectomy and liver metastases]. Gan To Kagaku Ryoho. 2011;38(12):2369-2371.

9. Farhat MH, Shamseddine AI, Barada KA. Small bowel tumors: clinical presentation, prognosis, and outcome in 33 patients in a tertiary care center. J Oncol. 2008;2008:212067.

10. Krishnamurthy P, Varghese SE, Gopalswamy N, Hillman N, Ali SA. Small-bowel adenocarcinoma: case report and review of literature on diagnosis of small-bowel tumors. Gastroenterol Hepatol (N Y). 2007;3(2):129-135.

11. Higashi D, Ishibashi Y, Tamura T, Nii K, Egawa Y, Koga M, Tomiyasu T, et al. Clinical features of and chemotherapy for cancer of the small intestine. Anticancer Res. 2010;30(8):3193-3197.

12. Dasari BV, Gardiner KR. Management of adenocarcinoma of the small intestine. Gastrointest Cancer Res. 2009;3(3):121-122.

13. Nagaraj G, Zarbalian Y, Flora K, Tan BR, Jr. Complete response and prolonged disease-free survival in a patient with recurrent duodenal adenocarcinoma treated with bevacizumab plus FOLFOX6. J Gastrointest Oncol. 2014;5(1):E1-6. 